Research Paper

\title{
miR-155-5p Promotes Dorsal Root Ganglion Neuron Axonal Growth in an Inhibitory Microenvironment via the cAMP/PKA Pathway
}

\author{
Tianyi Wang1, ${ }^{*}$, Bo Li ${ }^{2,}$, , Zhijie Wang ${ }^{3,}$, , Xin Yuan ${ }^{4,}$, Chuanjie Chen ${ }^{5,}$, , Yanjun Zhang 4 , Ziwei Xia ${ }^{6}$, Xin
}

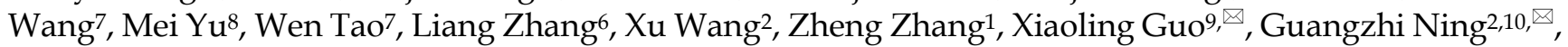

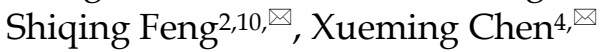

1. Department of Orthopedics, The 981st Hospital of the Chinese People's Liberation Army, Chengde 067000, Hebei Province, P.R. China

2. Department of Orthopedics, Tianjin Medical University General Hospital, Tianjin 300052, P.R. China

3. Department of Pediatric Internal Medicine, Affiliated Hospital of Chengde Medical University, Chengde 067000, Hebei Province, P.R. China

4. Department of Spine Surgery, Beijing Luhe Hospital, Capital Medical University, Beijing 100000, P.R. China

5. Department of Orthopedics, Chengde Central Hospital, Chengde 067000, Hebei Province, P.R. China

6. Department of Orthopedics, The Second Hospital of Tianjin Medical University, Tianjin 300211, P.R. China

7. Chengde Medical University, Chengde 067000, Hebei Province, P.R. China

8. Leukemia Center, Chinese Academy of Medical Sciences \& Peking Union of Medical College, Institute of Hematology \& Hospital of Blood Diseases, Tianjin 30020, P.R. China

9. Department of Neurology, The 981st Hospital of the Chinese People's Liberation Army, Chengde 067000, Hebei Province, P.R. China

10. Tianjin Neurological Institute, Key Laboratory of Post-Neuroinjury Neuro-repair and Regeneration in Central Nervous System, Ministry of Education and Tianjin City, Tianjin 300052, P.R. China

*Tianyi Wang, Bo Li, Zhijie Wang, Xin Yuan, Chuanjie Chen contributed equally to this article.

$\square$ Corresponding authors: Department of Neurology, The 981st Hospital of the Chinese People's Liberation Army, Chengde 067000, Hebei Province, P.R. China, E-mail address: 870808116@qq.com (X. Guo); Department of Orthopedics, Tianjin Medical University General Hospital, Tianjin 300052, P.R. China, E-mail address: wangxin_bao@sina.com (G. Ning); Department of Orthopedics, Tianjin Medical University General Hospital, Tianjin 300052, P.R. China, E-mail address: professorfengsq@163.com (S. Feng); Department of Spine Surgery, Beijing Luhe Hospital, Capital Medical University, Beijing 100020, P.R. China, E-mail address: liuyiyi@tmu.edu.cn (X.Chen).

(c) Ivyspring International Publisher. This is an open access article distributed under the terms of the Creative Commons Attribution (CC BY-NC) license (https://creativecommons.org/licenses/by-nc/4.0/). See http://ivyspring.com/terms for full terms and conditions.

Received: 2018.11.30; Accepted: 2019.04.29; Published: 2019.06.02

\begin{abstract}
Sensory dysfunction post spinal cord injury causes patients great distress. Sciatic nerve conditioning injury $(\mathrm{SNCl})$ has been shown to restore sensory function after spinal cord dorsal column injury (SDCL); however, the underlying mechanism of this recovery remains unclear. We performed a microarray assay to determine the associated miRNAs that might regulate the process of $\mathrm{SNCl}$ promoting SDCL repair. In total, 13 miRNAs were identified according to our inclusion criteria, and RT-qPCR was used to verify the microarray results. Among the 13 miRNAs, the miR-155-5p levels were decreased at $9 \mathrm{~h}, 3 \mathrm{~d}, 7 \mathrm{~d}, 14 \mathrm{~d}$, $28 \mathrm{~d}, 2 \mathrm{~m}$ and $3 \mathrm{~m}$ timepoints in the SDCL group, while the $\mathrm{SNCl}$ group had a smaller decrease. Thus, miR-155-5p was chosen for further study after a literature review and an analysis with the TargetScan online tool. Specifically, miR-155-5p targets PKI- $\alpha$, and the expression pattern of PKI- $\alpha$ was opposite that of miR-155-5p in both the SDCL and SNCl groups. Interestingly, miR-155-5p could promote dorsal root ganglion (DRG) neuron axon growth via the cAMP/PKA pathway and in a TNF- $\alpha$, IL-1 $\beta$ or MAG inhibitory microenvironment in vitro. Furthermore, miR-155-5p could regulate the cAMP/PKA pathway and promote sensory conduction function recovery post dorsal column injury as detected by NF-200 immunohistochemistry, somatosensory-evoked potentials, BBB scale and tape removal test. Collectively, our results demonstrated that miR-155-5p participates in the molecular mechanism by which $\mathrm{SNCl}$ promotes the repair of SDCL and that upregulated miR-155-5p can repair SDCL by enhancing DRG neuron axon growth via the CAMP/PKA pathway. These findings suggest a novel treatment target for spinal cord injury.
\end{abstract}

Key words: spinal cord dorsal column injury; microRNA; sensory function; cAMP/PKA; miR-155-5p 


\section{Introduction}

Sensation dysfunction, including paresthesia, dysesthesia, and chronic neuropathic pain, can appear within months following spinal cord injury (SCI) [1-5]. This torturous experience may induce severe impairments to the quality of daily life, to a greater degree than paralysis [6]. Additionally, sensory dysfunctions can affect cortical arousal and subsequently contribute to cognitive deficiency [7] and mental illness [8]. The disruption of ascending sensory conduction fibers located at the spinal cord dorsal column contributes to the blockage of somatosensory input to the central nerve system [6]. Central nervous system axons in mammals were once thought to be incapable of regeneration post lesion [9]. However, more recent evidence has demonstrated that sciatic nerve conditioning injury (SNCI, sciatic nerve transection one week prior to spinal cord dorsal column injury) can improve the intrinsic axon regenerative potential of primary sensory neurons. This potential enhances the corresponding ascending sensory conduction fiber regeneration post spinal cord dorsal column lesion (SDCL) [10]. Due to the unpredictability of SCI and clinical ethics, the application of SNCI cannot be performed clinically. Thus, uncovering the underlining mechanism of how SNCI promotes SDCL repair and developing an efficient treatment strategy are of great importance to improve SCI patient outcomes.

miRNAs are molecules that are indispensable upstream regulators and that can regulate gene expression at the posttranscriptional level [11-14]. Many miRNAs have been found in the mammalian nervous system, such as the brain, spinal cord and dorsal root ganglion (DRG), where they have key roles in neuronal physiological and pathophysiological processes $[15,16]$. MiRNAome is a high-throughput method that shows miRNA expression differences and can clarify the potential mechanism by which SNCI promotes the repair of the SDCL $[17,18]$.

miR-155-5p has been shown to have a pivotal role in immune responses and oncogenesis [19]. miR-155-5p can restore neural function after central nervous system (CNS) injury [20, 21]. However, miR-155-5p KO mice showed increased neurodegeneration and microgliosis, suggesting a neuroprotective effect of miR-155-5p in neuronal injury [21]. miR-155-5p possesses a neural protective role in the regulation of the neuroinflammation response and neurodegeneration post traumatic brain injury [21]. Fassi et al. demonstrated that miR-155-5p can target PKI-a [22]. Studies have widely demonstrated that PKI-a is the most potent heat-stable inhibitory substance to block protein kinase A (PKA) activity [23]. Therefore, PKI-a has the ability to terminate the cAMP signaling pathway, which is a pivotal pathway in the promotion of neuron axon regeneration [24].

In this research, we investigated the role of miR-155-5p in DRG neuron axon regeneration in vitro and in vivo to clarify the underlining mechanism by which SNCI promotes the repair of SDCL.

\section{Materials and Methods}

\section{Ethics statement}

DRG neurons were extracted from 10 neonatal Wistar rats $(<24 \mathrm{~h})$ and 204 adult female Wistar rats $(250 \pm 20 \mathrm{~g})$ for application in in vivo experiments, and all rats were purchased from the Animal Center of Radiation Medicine Institute (Tianjin, China). The rats were housed individually with food and drink ad libitum in an animal center maintained at stable humidity with a 12-hour light/dark cycle. All protocols were approved by the Animal Ethics Committee of the 266th Hospital of the Chinese People's Liberation Army (Approval No. 20160134). All protocols were conducted in accordance with the National Institutes of Health Guide for the Care and Use of Laboratory Animals (NIH Publications no. 85-23, revised 1996).

\section{Animal grouping}

To identify differentially expressed miRNAs in the SDCL and SNCI groups at $9 \mathrm{~h}, 3 \mathrm{~d}, 7 \mathrm{~d}, 14 \mathrm{~d}, 28 \mathrm{~d}$, $2 \mathrm{~m}$ and $3 \mathrm{~m}, 60$ Wistar rats were randomly divided into three groups for microarray analysis: four rats for the Sham group and 56 rats for the SDCL and SNCI groups (28 rats/group, 4 rats/timepoint). To demonstrate the expression pattern of miR-155-5p in the SNCI group resulting from both sciatic nerve transection and dorsal column lesion, 84 rats (4 rats/timepoint, 28 rats/group) in simple sciatic nerve injury (SSNI), SDCL and SNCI groups were used, and the expression variation of miR-155-5p was compared. Finally, to analyze the modulatory effect of miR-155-5p in vivo, 60 rats were randomly separated into Sham, SDCL, Ad-NC, Ad-miR and SNCI groups (12 rats/group).

\section{Sciatic nerve injury generation}

A sciatic nerve injury was generated in the SNCI and SSNI groups by cutting the sciatic nerve as previously described [17]. Briefly, the rats were anesthetized intraperitoneally with chloral hydrate $(10 \%, 0.3 \mathrm{ml} / 100 \mathrm{~g})$. After sterilizing the skin, the bilateral sciatic nerves were exposed, and $0.2 \mathrm{~cm}$ of sciatic nerve that was $1.5 \mathrm{~cm}$ caudal to the infrapiriform foramen was removed; then, the incisions were closed. Subutex $(50 \mu \mathrm{g} / \mathrm{kg})$ was used to 
relieve pain once per day for three days.

\section{Dorsal column lesion generation}

To generate a spinal cord dorsal column injury in the SDCL and SNCI groups, the spinal cords of the rats were injured as previously described [17]. Briefly, after satisfactory anesthetization, the dorsal side of the spinal cord at the T10 level was exposed, and the theca vertebralis was cut carefully without damaging the spinal cord. Then, the dorsal side of the spinal cord $(0.2 \mathrm{~cm}$ in depth) between the dorsal roots was crush with fine watchmaker's forceps [25, 26]. The incision was then sutured tightly, and the rats were transferred to a warm pad to wake.

\section{Sample collection}

After anesthetization as described above, ice cold saline (200 $\mathrm{ml}$ per rat) and paraformaldehyde $(4 \%, 200$ $\mathrm{ml}$ per rat) was perfused trans-cardially. The spinal cords and DRG tissues were extracted at the indicated time points and frozen in liquid nitrogen, and then the samples were stored at $-80^{\circ} \mathrm{C}$ for subsequent microarray, RT-qPCR assay, immunohistochemistry staining and western blot analysis.

\section{Cell culture}

The underlying mechanism by which miR-155-5p regulates neuronal axon growth was assessed by extracting DRG neurons from neonatal Wistar rats and culturing them in vitro as previously described [27]. Briefly, after sterilizing the neonatal rats, DRG tissues near the spinal cord were extracted and washed with PBS. The extracted DRG tissues were separated mechanically into pieces and dissociated into single cells with trypsin $(0.125 \%)$ (Gibco, Grand Island, NY, U.S.). Then, the individual cells were seeded onto poly-L-lysine (Gibco, Grand Island, NY, U.S.)-coated plates for further study.

\section{Transfection}

The function of miR-155-5p was assessed in vitro with an oligonucleotide inhibitor and mimics transfected with Lipofectamine 3000 (Invitrogen, Carlsbad, USA) at the 4th day of primary DRG neuron culture to observe axon growth. All chemically modified (23-O-Methyl) oligonucleotides (microRNA155-5p mimic (5'-UUAAUGCUAAUCGUGAUAG GGGU-3') and microRNA-155-5p inhibitor (5'-ACCCCUAUCACGAUUAGCAUUAA-3')) were purchased from GenePharma [28]. At $24 \mathrm{~h}$ after transfection, the DRG neuron culture medium was changed to complete culture medium.

\section{Drug treatment}

After culturing the DRG neurons for four days, the cells were treated with IL-1 $\beta(40 \mathrm{ng} / \mathrm{ml})$ [29],
TNF-a (100 ng/ml) (Cell Signaling, Danvers, MA) [30], H89 (10 $\mu \mathrm{M})$ (Sigma-Aldrich, St. Louis, USA) [31, 32] or MAG-FC $(25 \mu \mathrm{g} / \mathrm{ml}, \quad \mathrm{R} \& D$ Systems, Minneapolis, USA)[33]; then, neurite length was assessed.

\section{RT-qPCR}

The expression of miRNAs and PKI- $\alpha$ mRNA was detected using a mirVanaTM RNA Isolation kit (Invitrogen, Carlsbad, USA) to extract total RNA from cultured DRG neurons and DRG tissues. cDNA synthesis was conducted with a miScript II Reverse Transcription kit (Qiagen, Hilden, Germany) or PrimerScript RT reagent kit (Takara Bio, Inc., Otsu, Japan) for miRNA and mRNA detection, respectively. PCR reaction mixtures $(20 \mu \mathrm{l})$ for miRNA or mRNA detection were tested using a LightCycler ${ }^{\circledR} 480$ II Real-time PCR Instrument (Roche Diagnostics, Basel, Switzerland) with miScript SYBR® Green PCR Kit (QIAGEN, Dusseldorf, Germany). The primers were listed as following:

PKI-a: F: 5'-AGACAGAAGGTGAAGATGAT GG-3';

R: 5'-AGCAATGCCAGGAGATTCG-3'; TGA-3';

miR-155-5p: F: 5'-GGCCCTTTAATGCTAATCG

R: 5'-GTCTCCTCTGGTGCAGGGTCCGAGGTA TTCGCACCAGA GGAG ACACCCCT-3';

GAPDH: F: 5'-GCACCGTCAAGGCTGAGA AC-3';

R: 5'-TGGTGAAGACGCCAGTGGA-3';

U6: F: 5'-CAGCACATATACTAAAATTGGAA CG-3';

R: 5'-ACGAATTTGCGTGTCATCC-3'.

The expression of miRNAs and mRNA was standardized to U6 or GADPH mRNA expression, respectively. The experiments were conducted at least three times [34].

\section{ELISA}

The expression profile of IL-1 $\beta$ and TNF- $\alpha$ in the spinal cord was determined by ELISA [35], and sample preparation was conducted according to the manufacturer's instructions. Subsequently, TNF-a and IL-1 $\beta$ content in the sample supernatant was detected with IL-1 $\beta$ and TNF- $\alpha$ ELISA kits (R\&D Systems, Minneapolis, MN, USA), respectively. Each test was repeated three times.

\section{Western blotting}

To detect the expression of associated proteins in DRG tissues and cultured DRG neurons, western blotting was conducted as previously described [36]. Briefly, the samples from cultured DRG neurons and DRG tissues from Wistar rats were harvested and lysed with RIPA buffer (Santa, Dallas, USA) 
supplemented with complete protease inhibitor (Sigma, St. Louis, USA). A Nanodrop 2000 (Thermo Scientific, Waltham, MA, USA) was used to measure the sample protein concentrations. The proteins in different samples were separated by electrophoresis via SDS-PAGE, and then the separated proteins were transferred to nitrocellulose membranes. After blocking with 5\% nonfat milk, the nitrocellulose membranes were probed with primary antibodies against PKI- $\alpha$ (Sigma- Aldrich, St Louis, USA), p-CREB (Santa, Dallas, USA), RhoA (Santa, Dallas, USA), Arg-1 (Abcam, Cambridge, UK) and GADPH (Proteintech, Chicago, USA). The membranes were incubated with a secondary horseradish peroxidase-conjugated antibody (Huaan, Hangzhou, China). The experiments were performed at least three times.

\section{Pull-down assay}

The expression of GTP-RhoA was detected by western blotting after pull-down assays (Upstate Biotechnology, NY, USA). For the pull-down assay, briefly, the samples from cultured DRG neurons and DRG tissues were collected and lysed in lysis/wash buffer (EMD Millipore, Darmstadt, Germany) on ice after washing with cold TBS. Then, the sample lysates were added to a RhoA activation assay reagent to bind the GTP-RhoA in the lysates. After incubating at $4^{\circ} \mathrm{C}$ for $45 \mathrm{~min}$ and centrifuging, the expression of GTP-RhoA was detected with an anti-RhoA antibody by western blot.

\section{Luciferase assay}

The 3'UTR fragment of PKI- a mRNA containing the potential binding site of miR-155-5p was inserted into a pMIR-REPORTTM luciferase reporter vector (Ambion, Austin, TX, USA), and the resulting plasmid was named PKI-a-WT. Using the same method, another vector containing a mutant PKI-a 3'UTR at the binding site of miR-155-5p was constructed and named PKI-a-MUT. The reconstructed reporter vectors (PKI-a-WT or PKI-a-MUT) were cotransfected with miR-155-5p or miR-NC into HEK293T cells. Luciferase activity was detected $24 \mathrm{~h}$ posttransfection with the Dual-Glo Luciferase Reporter Assay (Promega, USA, Madison) [22].

\section{DRG neuron neurite length detection}

Cultured DRG neuron cells were fixed, blocked and probed with an anti-NF200 antibody (Abcam, Cambridge, UK) and goat-anti-rabbit IgG-Cy5 (Abcam, Cambridge, UK), followed by DAPI (Sigma, St. Louis, MO, USA) staining. Fluorescence microscopy (Nikon TiU, Tokyo, Japan) was used for imaging, and the mean total length of the neurites extending directly from the cell body (100 neurons/well, 3 wells/group) was calculated using Image-Pro Plus 6.0 software (Media Cybernetics, Silver Spring, MD, USA).

\section{Adenovirus production and injection}

A reconstructed adenovirus of miR-155-5p was purchased from Yijun Co., Ltd. (Tianjin, China). Ad-miR-155-5p and Ad-NC were designed by the AdMax adenovirus system (Microbix Biosystems, Toronto, Canada). Then, the virus particles were amplified in HEK293A cells. After adenovirus preparation, the DRGs of rats from L4-L6 were surgically exposed; a glass needle with $1.1 \mu \mathrm{l}$ of adenovirus solution was inserted into the DRG tissue at a depth of $400 \mu \mathrm{m}$, held for 3 mins, injected an adenovirus solution $(0.2 \mu \mathrm{l} / \mathrm{min})$, and held for $5 \mathrm{~min}$ postinjection. Then, the incision was sutured loosely [37].

\section{Somatosensory evoked potentials (SSEP)}

The integrity of the sensory conductive pathway was assessed by SSEP [38]. Eight weeks post-SDCL, the SSEP assays were performed as described previously [38]. Briefly, stimulator electrode needles were punctured into the muscle near the rostral side of the severed sciatic nerve $0.5 \mathrm{~cm}$ distal to the infrapiriform foramen $(2.1 \mathrm{~mA}, 4.1 \mathrm{~Hz})$. The electrode recording needle was placed subcutaneously between the ears, and the electrode reference needle was placed subcutaneously at the root of the nose. A standard SSEP waveform contains a positive $\mathrm{P}$ peak and a negative $\mathrm{N}$ peak. An averaged waveform was calculated using 200 repeated stimulations. The consumed time from the start of stimulation to the appearance of the $\mathrm{N}$ peak was recorded as the $\mathrm{N}$-latency. The potential difference between $\mathrm{N}$ and $\mathrm{P}$ peaks was recorded as the N-P amplitude. The N-latency and N-P amplitude were analyzed to reflect the integrity of the sensory conductive pathway. The recorded data were averaged for the four rats in each group. Subsequently, the rats were sacrificed for immunohistochemistry analysis.

\section{Behavioral analysis}

To clarify the locomotor functional differences in the Sham, SDCL, Ad-NC, SNCI and Ad-miR treatment groups at the $1 \mathrm{~d}, 3 \mathrm{~d}, 1 \mathrm{w}, 2 \mathrm{w}, 3 \mathrm{w}, 4 \mathrm{w}, 5$ $\mathrm{w}, 6 \mathrm{w}, 7 \mathrm{w}$ and $8 \mathrm{w}$ timepoints, the Basso, Beattie, Bresnahan (BBB) Locomotor Rating Scale was applied. The sensory functional differences in the Sham, SDCL, Ad-NC, SNCI and Ad-miR groups at the $-1 \mathrm{w},-1 \mathrm{~d}, 1$ $\mathrm{w}, 2 \mathrm{w}, 3 \mathrm{w}, 4 \mathrm{w}, 5 \mathrm{w}, 6 \mathrm{w}, 7 \mathrm{w}$ and $8 \mathrm{w}$ timepoints were detected by a tape removal test (TRT). Locomotive function was assessed using the BBB Scale. The BBB scale ranges from 0 to 21 , in which 0 represents complete paralysis and 21 represents 
normal motor function [39]. After the BBB scale evaluation, sensory function was assessed by TRT. As reported previously, TRT is a sensitive test to assess sensory and motor function [40,41]. The tape was ensured not to spontaneously fall off and could be removed without adhering any hair. The data from both hindlimbs were recorded and averaged. Each rat was tested three times. The TRT baseline was recorded 1 week prior to the dorsal column injury. The time latency to sense the affixed tape $(0.9 \mathrm{~cm} \times 2.5$ $\mathrm{cm})$ and the removal latency were collected. If the sense latency time was over $120 \mathrm{~s}$, then $120 \mathrm{~s}$ was set as the time latency [40].

\section{Histological analysis}

Collected spinal cord and DRG tissue samples were subjected to immunohistochemistry or immunofluorescence staining. Briefly, after fixing with $10 \%$ paraformaldehyde, slicing horizontally (16 $\mu \mathrm{m})$, and blocking with $10 \%$ rabbit serum, the DRG samples were probed with anti-Neurofilament protein 200 (NF-200) (1:200; Abcam), goat anti-rabbit IgG/biotin (1:200; Abcam), and tertiary antibody streptavidin/HRP (1:300; Abcam). DAB and hematoxylin were applied to stain the slices. For spinal cord NF-200 immunofluorescence staining, anti- NF-200 (1:200; Abcam) and goat anti-rabbit IgG-cy3 (1:200; Abcam) were applied. The pictures were captured with a fluorescence microscope (Nikon TiU, Tokyo, Japan).

\section{Statistical analysis}

All data were analyzed using Graphpad Prism 6.0 software, and the results are presented as the mean + SD. One-way ANOVA followed by Tukey's post hoc multiple-comparisons tests were applied to analyze significant differences. Finally, $\mathrm{p}<0.05$ was considered to be statistically significant.

\section{Results}

\section{Characterization of miRNAs expressed by dorsal root ganglions of the SDCL and SNCI groups}

The in vivo miRNA expression profiles of DRGs of the SDCL and SNCI groups were assessed. Table 1 shows miRNAs with a fold change greater than 2 or less than -2 compared to the control group for at least three timepoints of the SDCL or SNCI group. Only miRNAs with biological functions in the central nervous system that have been previously validated in in vivo or in vitro studies were included. The identification of 13 relevant miRNAs was further validated by real-time PCR. Overall, the fold changes obtained by microarray and real-time PCR were similar for these miRNAs.

Table 1. The listed miRNAs based on inclusion criteria from microarray

\begin{tabular}{|c|c|c|c|c|c|c|c|c|c|}
\hline & group & $9 \mathrm{~h}$ & $3 \mathrm{~d}$ & $7 \mathrm{~d}$ & $14 \mathrm{~d}$ & $28 \mathrm{~d}$ & $2 \mathrm{~m}$ & $3 \mathrm{~m}$ & Function \\
\hline \multirow[t]{2}{*}{ rno-miR-142-3p } & SDCL & 1.56 & 2.56 & 3.34 & 4.19 & 4.7 & 4.78 & 4.85 & cell cycle and apoptosis [56] Glioblastoma multiforme [57] neuronal \\
\hline & SNCI & 1.07 & 1.27 & 1.53 & 1.8 & 2.06 & 2.17 & 2.33 & differentiation [58] neuroinflammation [59] axon growth [14] \\
\hline \multirow[t]{2}{*}{ rno-miR-155-5p } & SDCL & 0.63 & 0.53 & 0.45 & 0.26 & 0.24 & 0.22 & 0.22 & apoptosis[60] Glutamate toxicity [61] neuroinflammation [62] \\
\hline & SNCI & 0.92 & 0.89 & 0.77 & 0.73 & 0.52 & 0.47 & 0.47 & Immunomodulation [63] \\
\hline \multirow[t]{2}{*}{ rno-miR-17-5p } & SDCL & 0.85 & 0.33 & 0.35 & 0.35 & 0.35 & 0.35 & 0.35 & apoptosis[64] neuroinflammation [65] astrocyte proliferation [66] NPC \\
\hline & SNCI & 1.18 & 4.15 & 3 & 4.75 & 3 & 2.5 & 2 & differentiation [67] \\
\hline \multirow[t]{2}{*}{ rno-miR-500 } & SDCL & 0.76 & 0.51 & 0.67 & 0.69 & 0.49 & 0.43 & 0.32 & GABAergic dysfunction [68] \\
\hline & SNCI & 1.25 & 1.51 & 2.15 & 1.24 & 0.76 & 0.62 & 0.54 & \\
\hline \multirow[t]{2}{*}{ rno-miR-96 } & SDCL & 0.7 & 0.56 & 0.35 & 0.43 & 0.51 & 0.64 & 0.73 & autophagy [69] apoptosis [70]GSH regulation [71] neural induction [72] \\
\hline & SNCI & 2.15 & 2.51 & 3.13 & 0.65 & 0.53 & 0.41 & 0.32 & \\
\hline \multirow[t]{2}{*}{ rno-miR-129-1 } & SDCL & 0.8 & 0.5 & 0.4 & 0.3 & 0.3 & 0.3 & 0.3 & cell cycle [73] \\
\hline & SNCI & 1.58 & 1.21 & 2.52 & 2.51 & 3.56 & 2.56 & 3.51 & \\
\hline \multirow[t]{2}{*}{ rno-miR-136-5p } & SDCL & 0.38 & 0.39 & 0.42 & 0.45 & 0.52 & 0.61 & 0.76 & neuroinflammation [74] \\
\hline & SNCI & 0.41 & 0.42 & 0.21 & 0.15 & 0.45 & 0.47 & 0.67 & \\
\hline \multirow[t]{2}{*}{ rno-miR-15b } & SDCL & 1.27 & 1.3 & 1.4 & 1.8 & 2.9 & 3.6 & 4.16 & apoptosis $[75,76]$ cell cycle [77] neurogenesis [78] \\
\hline & SNCI & 0.87 & 0.67 & 1.22 & 1.21 & 1.22 & 1.23 & 1.24 & \\
\hline \multirow[t]{2}{*}{ rno-miR-181b-5p } & SDCL & 0.25 & 0.33 & 0.4 & 0.6 & 0.78 & 0.85 & 0.89 & astrocytoma [79] \\
\hline & SNCI & 1.23 & 2.21 & 2.65 & 2.23 & 1.24 & 1.23 & 0.99 & \\
\hline \multirow[t]{2}{*}{ rno-miR-21 } & SDCL & 0.32 & 0.43 & 3 & 3.5 & 4 & 4.3 & 4.3 & apoptosis [80] axongrowth [81] neuroinflammation [82] \\
\hline & SNCI & 3.51 & 3.51 & 2.51 & 2.15 & 1.51 & 1.24 & 1.23 & \\
\hline \multirow{2}{*}{ rno-miR-320a } & SDCL & 0.4 & 0.5 & 0.65 & 0.73 & 2.31 & 1.45 & 1.12 & $\mathrm{BSCB} / \mathrm{BBB}[83,84]$ \\
\hline & SNCI & 1.51 & 2.51 & 3.15 & 3.14 & 4.52 & 1.23 & 0.98 & \\
\hline \multirow[t]{2}{*}{ rno-miR-34c } & SDCL & 0.52 & 0.45 & 0.51 & 0.7 & 0.72 & 0.74 & 0.81 & PD pathogenesis [85] neurotoxicity [86] Glioma [87] AD [88] mitochondrial \\
\hline & SNCI & 3.22 & 2.32 & 2.13 & 2.01 & 1.79 & 1.65 & 1.21 & function [89] \\
\hline \multirow[t]{2}{*}{ rno-miR-431 } & SDCL & 1.2 & 1.51 & 1.65 & 1.21 & 0.97 & 0.51 & 0.62 & $\mathrm{~A} \beta$-toxicity $[90]$ neurite growth $[91]$ \\
\hline & SNCI & 2.31 & 3.21 & 4.12 & 0.83 & 0.64 & 0.32 & 0.12 & \\
\hline
\end{tabular}

SDCL: spinal cord dorsal column injury; SNCI: sciatic nerve conditioning injury; AD: Alzheimer's Disease; PD: Parkinson's disease; BSCB: blood spinal cord barrier; BBB: blood brain barrier; GSH: Glutathione; NPC: neural precursor cells 
A

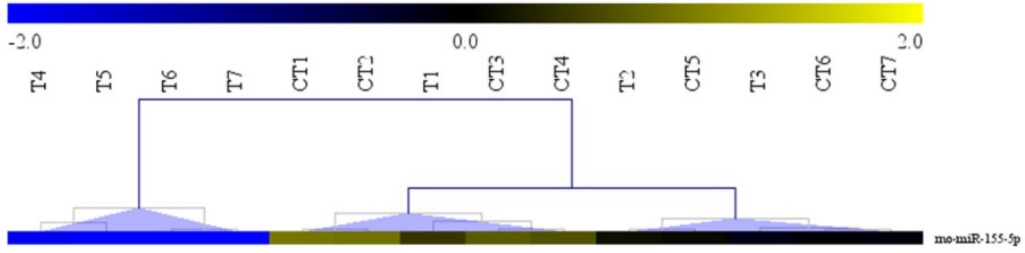

B

\begin{tabular}{ll}
\hline & $\begin{array}{l}\text { Predicted consequential pairing of target region } \\
\text { (top) and miRNA (bottom) }\end{array}$ \\
\hline Position 443-449 of PKIA 3' UTR & $5^{\prime}$...CCAGAGAGCACAGUUAGCAUUAU... \\
rno-miR-155-5p & $3^{\prime} \quad$ UGGGGAUAGUGUUAAUCGUAAUU \\
\hline
\end{tabular}

Fig. 1. Hierarchical clustering analysis and the predicted target gene of miR-155-5p. (A) Hierarchical clustering analysis results of miR-155-5p in the SDCL and SNCl groups (T1 T7: $9 \mathrm{~h}, 3 \mathrm{~d}, 7 \mathrm{~d}, 14 \mathrm{~d}, 28 \mathrm{~d}, 2 \mathrm{~m}$, and $3 \mathrm{~m}$ post spinal cord dorsal column lesion in the SDCL group; CTI CT7: $9 \mathrm{~h}, 3 \mathrm{~d}, 7 \mathrm{~d}, 14 \mathrm{~d}, 28 \mathrm{~d}, 2 \mathrm{~m}$, and $3 \mathrm{~m}$ post spinal cord dorsal column lesion in the SNCl group). (B) The potential binding sites of miR-155-5p and the PKI- $\alpha$ mRNA 3'UTR.

A

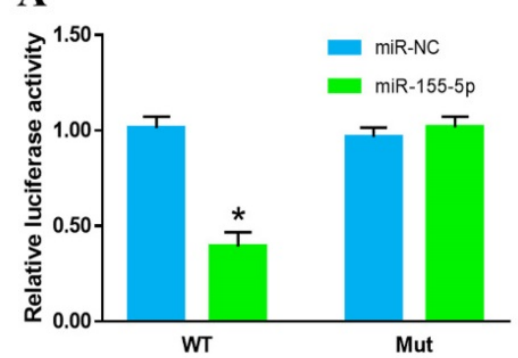

B

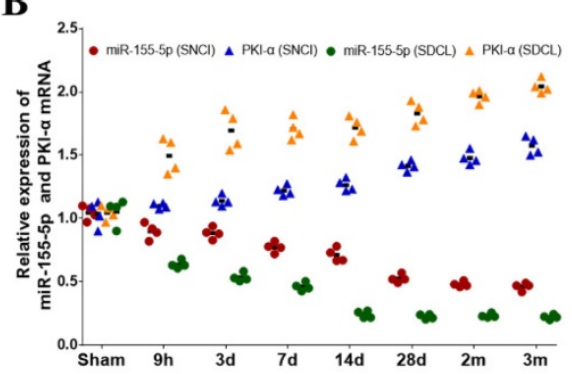

C

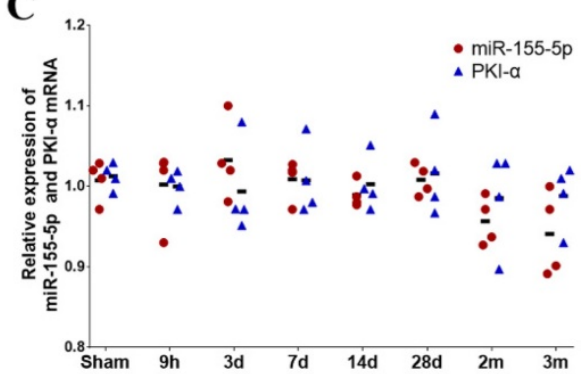

Fig. 2. Dual-luciferase reporter assay and the expression of PKI- $\alpha$ mRNA and miR-155-5p in the SDCL, SNCI and SSNI groups. (A) Dual-luciferase reporter assay (WT: wild type; MUT: mutant). (B) The expression of PKI- $\alpha$ mRNA and miR-155-5p in the SDCL and SNCI groups. (C) The expression of PKI- $\alpha$ mRNA and miR-155-5p in the SSNI group.

In the miRNAs in Table 1, miR-155-5p showed a tendency to decrease gradually at the $9 \mathrm{~h}, 3 \mathrm{~d}$, and $7 \mathrm{~d}$ timepoints and then decrease sharply from $14 \mathrm{~d}$ to $3 \mathrm{~m}$ in the SDCL group. In the SNCI group, miR-155-5p always showed a relatively gentle downward trend. Notably, at the last four timepoints, miR-155-5p in the SDCL group was downregulated more than 2-fold compared with the SNCI group. Hierarchical clustering results showed that the expression of miR-155-5p at $14 \mathrm{~d}, 28 \mathrm{~d}, 2 \mathrm{~m}$ and $3 \mathrm{~m}$ in the SDCL group formed a cluster. Meanwhile, the miR-155-5p expression patterns for the other timepoints of the SDCL and SNCI groups formed another cluster (Fig 1 A). After reviewing the literature and searching the TargetScan database, the target gene of miR-155-5p was hypothesized to be PKI- $\alpha$ (Fig 1 B), indicating the potential role of miR-155-5p in regulating neuron axon growth. Thus, the differential expression of miR-155-5p between the SDCL and SNCI groups may hint at one of the underlying mechanisms by which SNCI repairs a spinal cord dorsal column lesion. Thus, further research on miR-155-5p was warranted.

\section{miR-155-5p targets PKI- $\alpha$ mRNA}

To confirm the interaction between miR-155-5p and PKI- $a$, a dual-luciferase reporter assay was conducted. The reporter vectors were cotransfected with either miR-155-5p mimics or miR-NC, and the luciferase activities were detected. miR-155-5p mimics evidently inhibited the luciferase activity in PKI-a-WT-transfected cells, while there were no differences in the other three groups (Fig 2 A). These data indicated that miR-155-5p can directly interact with the 3'UTR of PKI-a mRNA. The expression of PKI- $\alpha$ mRNA in the SDCL and SNCI groups at $9 \mathrm{~h}, 3$ $\mathrm{d}, 7 \mathrm{~d}, 14 \mathrm{~d}, 28 \mathrm{~d}, 2 \mathrm{~m}$, and $3 \mathrm{~m}$ was detected. The RT-PCR results showed that the expression of PKI-a mRNA was opposite that of miR-155-5p in both groups (Fig 2 B).

\section{The expression pattern of miR-155-5p in the SNCI group depends on cooperation from SNCl and the dorsal column lesion}

SSNI was assessed to determine if it can induce a similar expression pattern of miR-155-5p in the SNCI group. The expression of miR-155-5p and PKI- $\alpha$ mRNA in the SSNI group was detected by RT-PCR (Fig 2 C). Our results indicated that SSNI did not change the expression of miR-155-5p. As shown above, miR-155-5p had a different expression trend between the SDCL and SNCI groups. These results demonstrated that the expression of miR-155-5p in 
SNCI resulted from the cooperation of both SNCI and SDCL. The expression trend of PKI-a was inversely correlated to that of miR-155-5p in all groups.

\section{miR-155-5p regulates DRG neuron axon growth via the CAMP/PKA pathway}

As shown above, PKI- $a$, the intrinsic inhibitor of PKA, is the target gene of miR-155-5p. Therefore, miR-155-5p could be an upstream regulator that modulates axon growth via the cAMP/PKA pathway. Cultured DRG neurons were treated with miR-155-5p mimics or inhibitor, and then the neurite length and the expression of PKI- $\alpha, p-C R E B, A r g-1$ and GTP-RhoA were assessed.

miR-155-5 $p$ mimics elongated the neuron axon, while the miR-155-5p inhibitor decreased the neurite length compared with the blank group. H89, a PKA pathway inhibitor, significantly decreased the axon length that was elongated by miR-155-5p mimics. These data indicated that miR-155-5p can enhance axon length via the cAMP/PKA pathway (Fig 3 A, B).

Furthermore, key proteins in the cAMP/PKA pathway were assessed (Fig $3 \mathrm{C}-\mathrm{G}$ ). The expression of PKI- $a$ was noticeably decreased in both the miR-155-5p mimic and miR-155-5p mimic\&H89 groups without a significant difference, while the expression was increased in the miR-155-5p inhibitor group compared with that in the blank group. The expression of p-CREB and Arg-1 was increased after miR-155-5p mimic treatment, while the miR-155-5p inhibitor inhibited the expression compared with that in the blank group. However, H89 significantly reversed the p-CREB and Arg-1 expression enhanced by miR-155-5p mimics. The expression of GTP-RhoA was inhibited in the miR-155-5p mimic group, while the miR-155-5p inhibitor significantly promoted GTP-RhoA expression compared with that in the blank group. H89 significantly reversed the inhibitory effect of miR-155-5p mimics on GTP-RhoA expression. $\mathrm{H} 89$ alone did not change the expression of PKI- $a$, but it evidently decreased the expression of p-CREB and Arg-1 and increased the expression of GTP-RhoA. These data indicated that miR-155-5p can regulate axon growth via the $\mathrm{CAMP} / \mathrm{PKA}$ pathway.

\section{miR-155-5p promotes DRG neuron axon growth in an inhibitory microenvironment}

The inhibitory microenvironment formed post spinal cord injury is a major aspect that inhibits neuronal axon regeneration. IL-1 $\beta$, TNF- $\alpha$ and MAG are three of the major inhibitory factors that interact with the RhoA/ROCK pathway to inhibit neuronal axon growth [42-44]. We wondered whether miR-155-5p could assist axon growth in an inhibitory microenvironment. DRG neurons showed a shorter axon in the IL-1 $\beta$, TNF- $\alpha$ and MAG groups compared with that in the blank group, while miR-155-5p mimics significantly reversed the inhibitory effect of IL-1 $\beta$, TNF- $\alpha$ and MAG. These results indicated that miR-155-5p can enhance DRG neuron axon growth in an inhibitory microenvironment that contains IL-1 $\beta$, TNF-a or MAG (Fig 4 A, B).
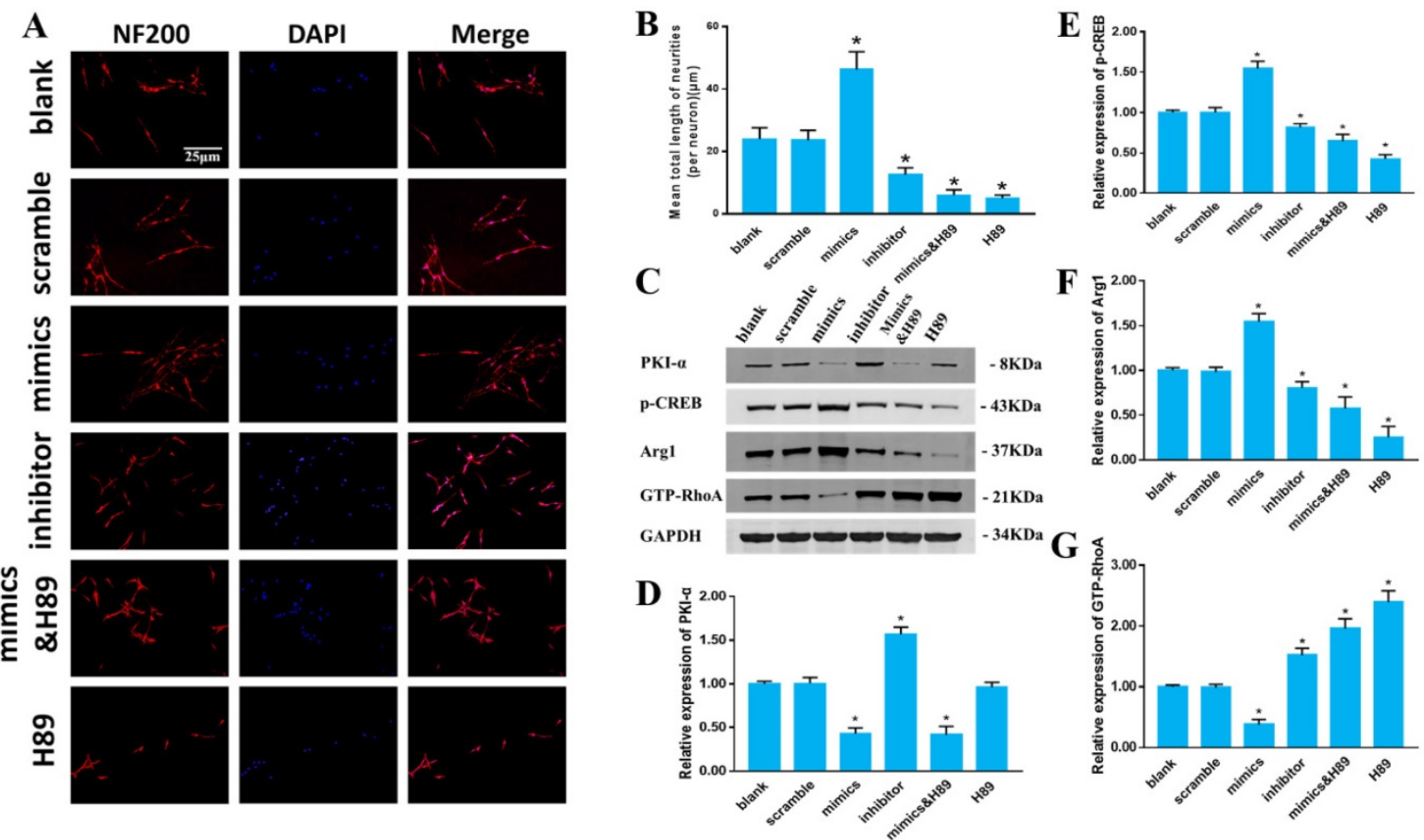

Fig. 3. miR-155-5p regulates DRG neuron axon growth via the cAMP/PKA pathway. (A) Immunofluorescent images of cultured DRG neurons. NF-200 staining is shown in red, and DAPI staining of the nuclei is shown in blue. (B) Quantitative histogram of the mean total neurite length (per neuron). (C) Relative protein expression of PKI-a, $\mathrm{P}-\mathrm{CREB}, \mathrm{Arg}-1$ and GTP-RhoA detected by western blot and normalized to GAPDH. (D-G) Quantitative histogram of the western blot. Data were expressed as the mean \pm SD of three experiments $(n=4, * P<0.05)$ 
A

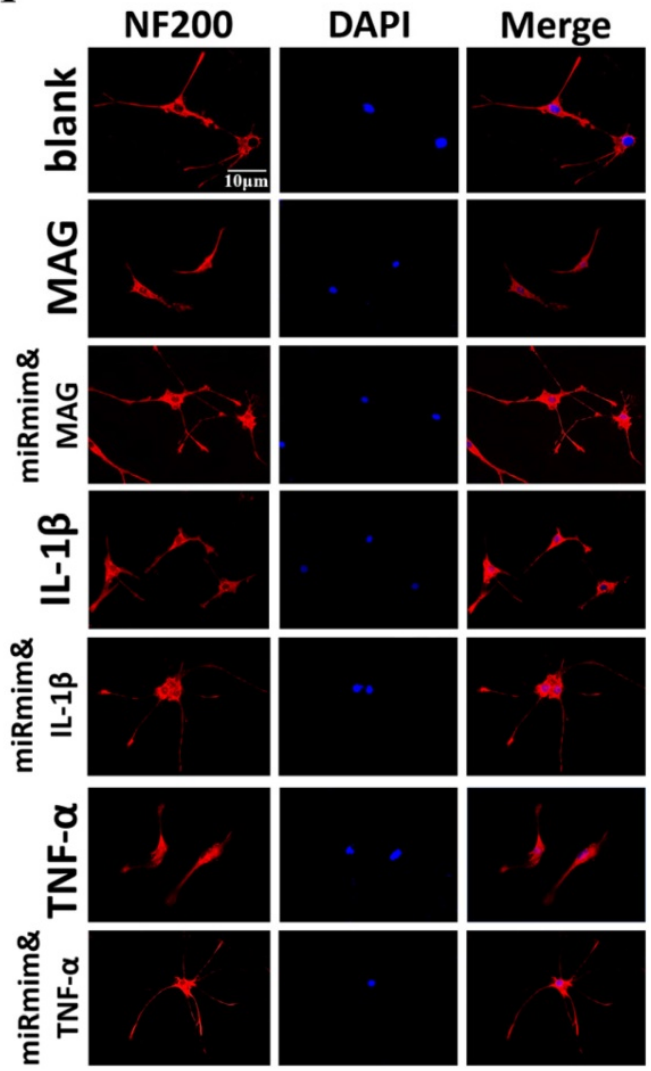

B

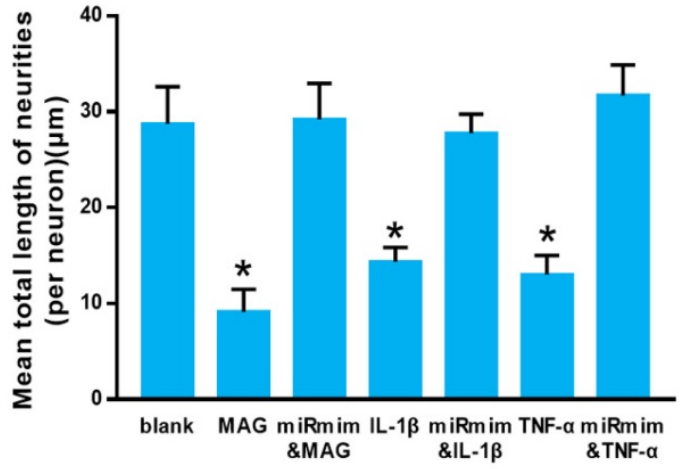

Fig. 4. miR-155-5p promotes DRG neuron axon growth in an inhibitory microenvironment. Immunofluorescent images of cultured DRG neurons. NF-200 staining is shown in red, and DAPI staining of nuclei is shown in blue. (B) Quantitative histogram of mean total neurite length (per neuron). Data are expressed as the mean \pm SD of three experiments $(n=4, * P<0.05)$

\section{miR-155-5p regulates the cAMP/PKA pathway in vivo}

To clarify whether miR-155-5p regulates the cAMP/PKA pathway in vivo, eight weeks after dorsal column injury, the expression of miR-155-5p, PKI-a, p-CREB, Arg-1 and GTP-RhoA was assessed in the Sham, SDCL, Ad-NC, Ad-miR and SNCI groups eight weeks after dorsal column injury. The expression of miR-155-5p was decreased in the SDCL and Ad-NC groups, and SNCI and Ad-miR weakened the decrease by SDCL compared with that in the Sham group (Fig 5 A). Compared with the Sham group, the expression of PKI- $\alpha$ was increased in the SDCL and Ad-NC groups. However, the expression of PKI-a was decreased in the SNCI and Ad-miR groups compared with that in the SDCL group. The expression of p-CREB and Arg-1 was significantly decreased in the SDCL and Ad-NC groups compared with that in the Sham group. SNCI and Ad-miR increased the expression of p-CREB and Arg-1 compared with that in the SDCL group. The expression of GTP-RhoA was increased in the SDCL and Ad-NC groups compared with that of the Sham group, while SNCI and Ad-miR suppressed these increases (Fig 5 B-F). These results indicated that
Ad-miR and SNCI can regulate the cAMP/PKA pathway via miR-155-5p in vivo.

\section{miR-155-5p does not improve the inhibitory microenvironment caused by dorsal column injury}

As demonstrated previously, SNCI can promote sensory conduction functional recovery [17]. However, whether this promotion is partly due to the improved inhibitory microenvironment at the injured site remains to be clarified. IL- $1 \beta$, TNF- $\alpha$ and MAG are three major inhibitory factors that interact with the RhoA/ROCK pathway to inhibit neuronal axon growth. MAG is a cell structure protein that is located on the periaxonal myelin membrane, and its expression decreases post spinal cord injury because of the damaged myelinated axon [45]. The expression of MAG was associated with the severity of injury other than the degenerated axon. The inhibitory effect of MAG on neurites is the result of the released MAG from damaged myelin cells rather than the expression level. However, as inflammatory factors, the expression of TNF- $\alpha$ and IL-1 $\beta$ was upregulated post spinal cord injury [5]. Therefore, the expression of TNF- $\alpha$ and IL-1 $\beta$ was detected in the Sham, SDCL, Ad-NC, Ad-miR and SNCI groups by ELISA. The 
expression of TNF- $\alpha$ and IL-1 $\beta$ was upregulated with no significant differences in the SDCL, Ad-NC, Ad-miR and SNCI groups compared with that in the Sham group (Fig 6 A, B). These results indicated that SNCI and Ad-miR treatment had no effect on improving the partly inhibitory microenvironment formed by TNF- $\alpha$ and IL-1 $\beta$ at the injured site of the dorsal column.

\section{miR-155-5p promotes sensory conduction function recovery post SDCL}

To determine the beneficial effect of miR-155-5p on dorsal column injury, NF-200 was detected in both DRG tissues and dorsal column tissues. Compared with the Sham group, the NF-200-positive area in both DRG tissues and dorsal column tissues was decreased significantly in the SDCL and Ad-NC groups. However, SNCI and Ad-miR improved the
NF-200-positive area compared with that in the SDCL group (Fig 7 A, B).

Furthermore, the sensory conductive function was assessed using SSEP, a widely applied method to reflect the integration of the sensory conductive pathway that originates from the peripheral nervous system to the brain. A normal SSEP waveform consisting of a positive $\mathrm{P}$ peak and a negative $\mathrm{N}$ peak was obtained from the Sham group, while there was no wave with $\mathrm{N}$ or P peaks obtained from the SDCL and Ad-NC groups, indicating that the sensory conductive pathway was totally destroyed in the SDCL and Ad-NC groups. However, a restored SSEP waveform with $\mathrm{N}$ and $\mathrm{P}$ peaks was obtained for the Ad-miR and SNCI groups, although the $\mathrm{N}$ peak latency and N-P amplitude were poorer than those of the Sham group (Fig 7 C-E).
A

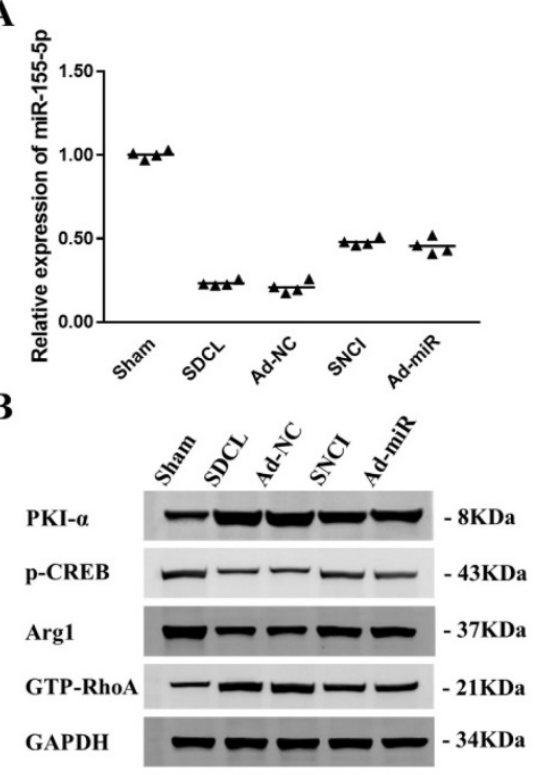

C

D
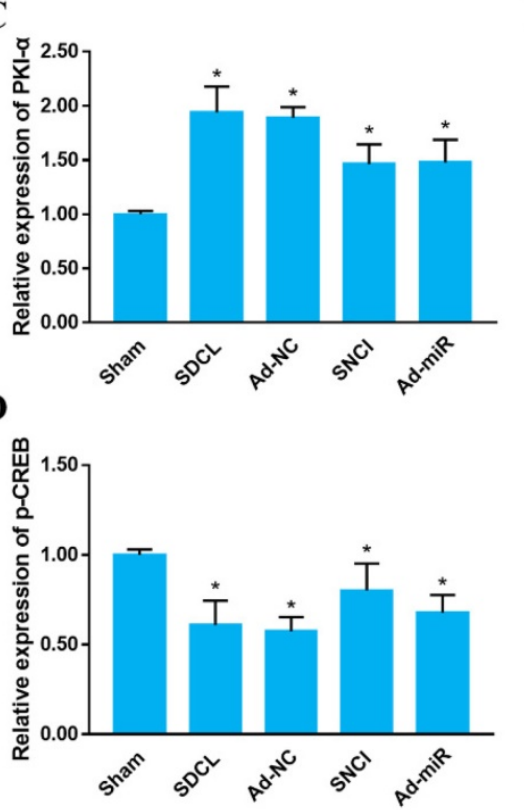

$\mathbf{E}$

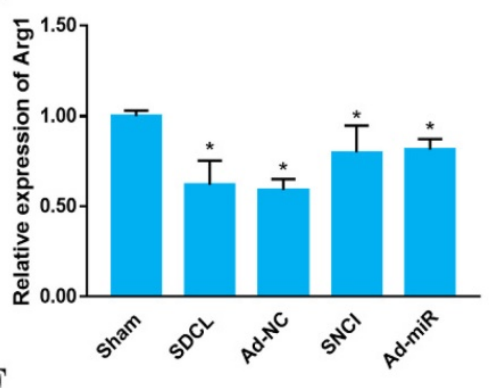

F

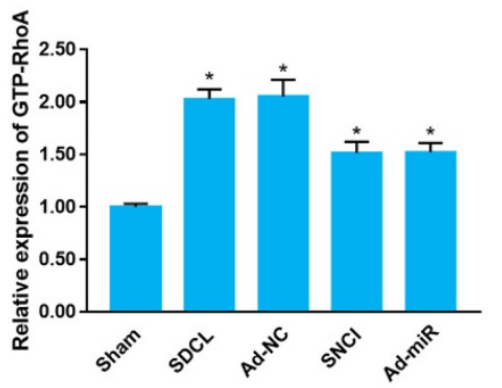

Fig. 5. miR-155-5p regulates the cAMP/PKA pathway in vivo. (A) Relative expression of miR-155-5p in DRG tissues post dorsal column injury detected by $R T-P C R$ ( $=4$, *P $<0.05$ ). (B) Relative protein expression of PKI- $\alpha$, p-CREB, Arg-1 and GTP-RhoA detected by western blot and normalized to GAPDH. (C-F) Quantitative histogram of the western blot. Data are expressed as the mean \pm SD of three experiments $(n=4, * P<0.05)$.

A

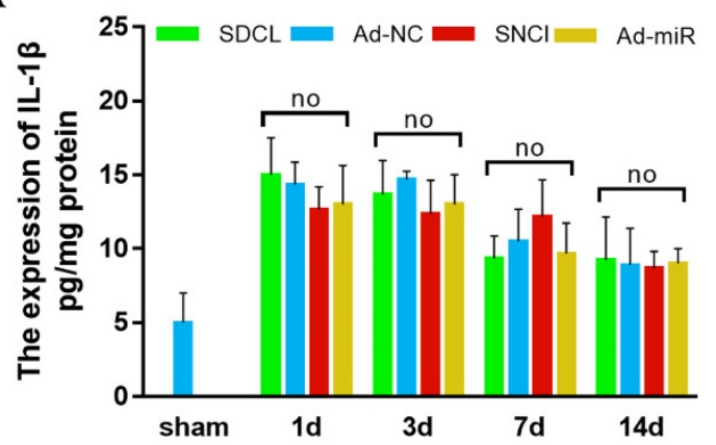

B

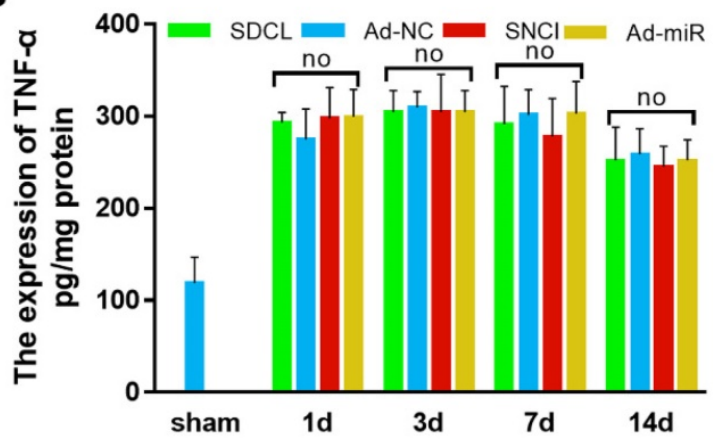

Fig. 6. Expression of TNF- $\alpha$ and IL-1 $\beta$. (A) IL-1 $\beta$ expression detected by ELISA. (B) TNF- $\alpha$ expression detected by ELISA. Data are expressed as the mean \pm SD of three experiments $(n=4, * P<0.05)$. 

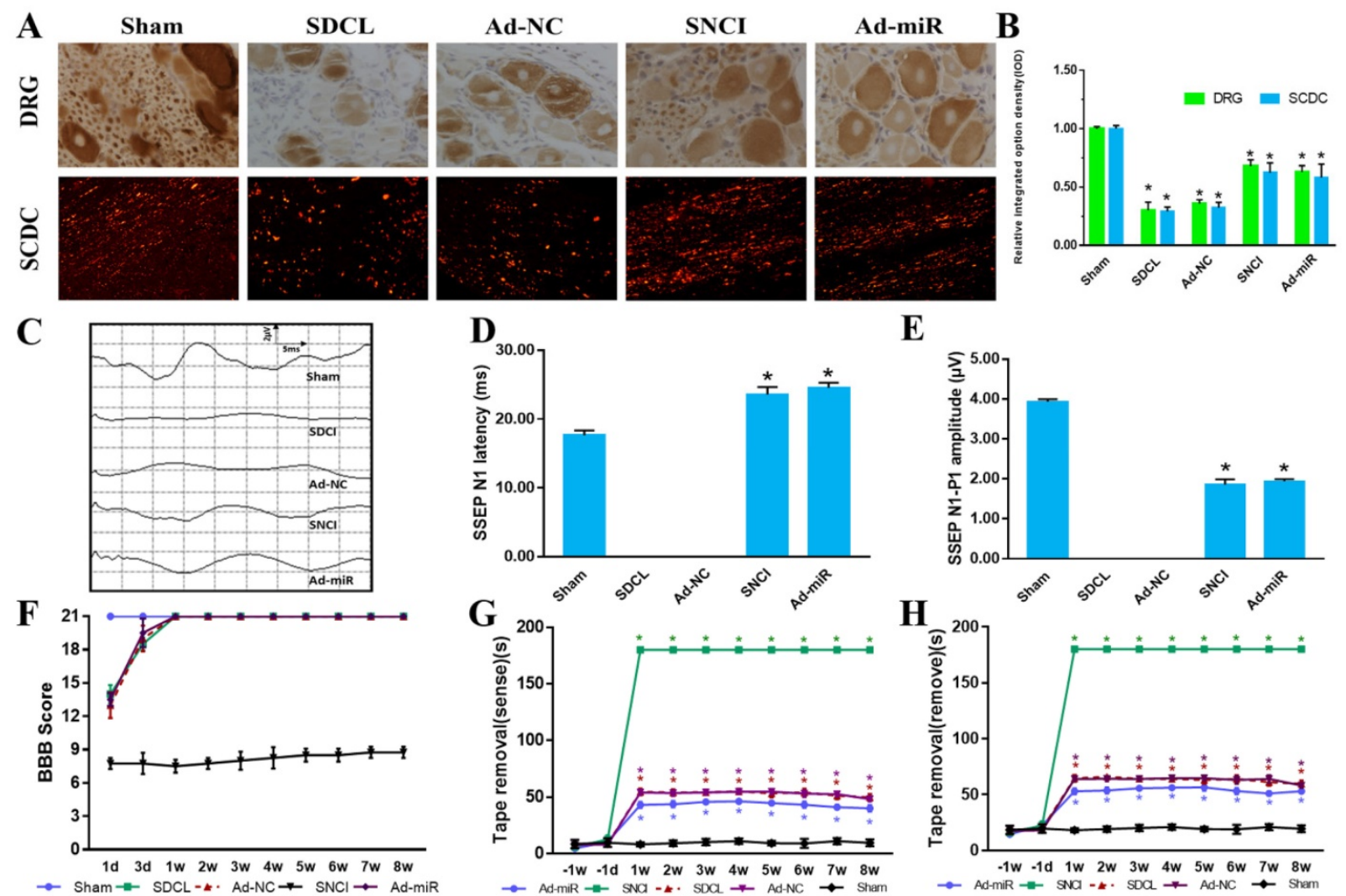

Fig. 7. miR-155-5p promotes sensory conduction function recovery post SDCL. NF-200 immunohistochemistry staining of DRG tissues (top panel) and NF-200 immunofluorescence staining of spinal cord dorsal column (bottom panel). (B) Relative integrated option density quantitative histogram of NF-200-positive images. (C) Representative average waveform curves of SSEP examination. (D) N latency quantitative histogram of SSEP. (E) N-P amplitude quantitative histogram of SSEP. (F) BBB scores. $(G, H)$ The latency of sensation and removal of the tape removal test. Data are expressed as the mean $\pm S D$ of three experiments $(n=4$, $* P<0.05)$.

We better clarified the sensory conductive function recovery using the BBB scale and TRT. As shown in Fig $7 \mathrm{~F}$, compared to the Sham group, the BBB scores in the SDCL, Ad-NC and Ad-miR groups decreased post dorsal column injury at $1 \mathrm{~d}$. However, the BBB scores in all groups returned to 21 after the 3 $\mathrm{d}$ time point. As the sciatic nerves were transected in the SNCI group, the BBB scores were decreased and sustained at a low level for all timepoints. The BBB score results indicated the success of the dorsal column injury model without damaging the locomotive function. For TRT, the time required for the rats to sense and remove the tape was recorded and analyzed (Fig. 7 G, H). Rats in the Sham group showed facile motion in removing the tape, whereas the removal latency times of rats in the Ad-NC and SDCL groups were delayed with no significant difference between them ( $P>0.05)$. Rats in the Ad-miR group showed a better performance in the latency times of the sensation and removal of the tape compared to those in the SDCL group $(\mathrm{P}<0.05)$. Because of the transected sciatic nerve in the SNCI group, the latency times were $180 \mathrm{~s}$ along the timepoints. However, the latency times for the rats in the Ad-miR group were still poorer than those in the Sham group $(\mathrm{P}<0.05)$. These results indicated that
miR-155-5p plays a critical role in DRG neuron axon growth and sensory conductive functional recovery post spinal cord dorsal column injury.

\section{Discussion}

Sensory dysfunction is one of the major complications of spinal cord injury. Distinct from motor conduction axons that move downward from brain in the ventral column of spinal cord, sensory conduction axons originate in the DRG and move upward in the dorsal column of the spinal cord. In addition to damage to locomotor function, sensory dysfunction below the injured level often affects cortical arousal and contributes to cognitive deficiencies [46] and psychological problems [47]. Unlike the peripheral nervous system, injured neuron axons in the central nervous system can hardly regenerate because of poor intrinsic regenerative ability, demyelination, inhibitory molecules (TNF-a, IL-1 $\beta$, MAG, and Nogo), glial scars, and cavity formation $[5,48]$. Previous studies have shown that a sciatic nerve lesion prior to spinal cord injury can promote DRG neuron central axon growth, which provides a potential method to restore sensory function [9]. However, the unpredictability of spinal cord injury and the ethical impossibility presented by 
sciatic nerve conditioning injury prevents its application in the clinic. Due to the ambiguity of the underlying mechanism of SNCI, an effective alternative strategy has yet to be developed.

Remarkably, miRNAs contribute to complex pathophysiological processes of spinal cord injury and other CNS insults [49-51]. A study in the New England Journal of Medicine showed that miRNA-122 is involved in a clinical trial for hepatitis $C$ virus infection [52], indicating the possibility of treating clinical diseases via targeting miRNAs. This study aimed to determine the key miRNAs to regulate the promotion of spinal cord sensory function recovery by SNCI and clarify the associated pathway in vitro and in vivo to develop an alternative treatment strategy.

To determine the underlying mechanism by which SNCI promotes dorsal column injury recovery, the miRNA expression profile of SDCL and SNCI group rats were detected by microarray. We found that miR-155-5p was downregulated at all time points in both groups. Remarkably, the SNCI group had a smaller decrease, which indicated the involvement of miR-155-5p in the promotion of dorsal column injury recovery by SNCI. To confirm our hypothesis, PKI-a, the target gene of miR-155-5p, was validated by TargetScan, an online miRNA targeting tool, literature research and dual-luciferase assay. Therefore, the potential modulatory role of miR-155-5p in DRG neuron axon growth was clarified.

Additionally, miR-155-5p exerted a beneficial effect on axon growth via the cAMP/PKA pathway by targeting the intrinsic PKA inhibitor, PKI- $a$. Furthermore, miR-155-5p promoted DRG neuron axon growth in a negative environment composed of TNF- $\alpha$, IL-1 $\beta$ or MAG. Moreover, the modulatory effect of miR-155-5p on the cAMP/PKA pathway and neuron axon growth in vivo were verified. Our in vivo data indicated that miR-155-5p has a beneficial role in promoting spinal cord sensory function recovery. The expression of TNF- $\alpha$ and IL-1 $\beta$ was similar in the SDCL and SNCI groups, which indicated that SNCI enhanced the neuronal intrinsic growth ability to promote sensory function recovery rather than improving the native negative microenvironment.

The consistent upregulation of miR-155-5p expression after controlled cortical impact demonstrated the important role of miR-155-5p [21]. Additionally, miR-155-5p KO mice showed increased neurodegeneration and microgliosis, indicating a neuroprotective role for miR-155 in neuronal injury [21]. Similarly, miR-155-5p can regulate PKA signaling via targeting an intrinsic inhibitor of PKA, PKI-a [19]. PKA is a major regulator in the nervous system, including neural development, axon growth, and behavior formation [53]. As an intrinsic inhibitor of PKA by inhibiting the C subunits, PKI-a plays a critical role in the cAMP/PKA pathway [54]. The cAMP/PKA pathway directly controls axon growth, and CREB is a key downstream transcription factor in the CAMP/PKA pathway that is associated with axon growth [23, 24], while the RhoA/ROCK pathway, downstream of cAMP/PKA, can be triggered by MAG [42], IL-1 $\beta$ [43] and TNF- $\alpha$ [44] and negatively control axon growth. The inhibition of TNF-a expression promotes Arg 1 expression [55]. Furthermore, Arg 1 improves axon growth via inducing polyamine expression, which directly controls cytoskeleton assembly and induces axon growth on MAG [14]. Therefore, miR-155-5p can regulate neuronal axon growth via modulating the cAMP/PKA pathway by targeting PKI-a in spinal cord dorsal column injury.

In conclusion, we demonstrated that miR-155-5p plays a pivotal role in controlling DRG neuron axon growth via the cAMP/PKA pathway, therefore improving sensory functional recovery post SDCL. Our findings suggest that miR-155-5p could be a novel therapeutic target to promote sensory functional recovery post SDCL.

\section{Conclusion}

In this study, miR-155-5p was identified to be a key regulator in the underlying mechanism of sciatic nerve conditioning injury that promotes spinal cord dorsal column injury recovery. miR-155-5p can enhance DRG neuron axon growth in an inhibitory microenvironment via the cAMP/PKA pathway by targeting PKI- $a$ in vitro and in vivo and promote the sensory function recovery after spinal cord dorsal column injury.

\section{Abbreviations}

Sciatic nerve conditioning injury (SNCI); spinal cord dorsal column injury (SDCL); simple sciatic nerve injury (SSNI); spinal cord injury (SCI); central nervous system (CNS); dorsal root ganglion (DRG); cyclic adenosine monophosphate (cAMP); protein kinase A (PKA); tumor necrosis factor- $\alpha$ (TNF- $\alpha$ ); Interleukin 1 beta (IL-1 $\beta$ ); myelin associated glycoprotein (MAG); Basso, Beattie and Bresnahan locomotor scale (BBB scale); KO (knock out); adenovirus (Ad); negative control (NC); Real-time quantified polymerase chain reaction (RT-qPCR); Glyceraldehyde 3-phosphate dehydrogenase (GAPDH); enzyme-linked immunosorbent assay (ELISA); sodium dodecyl sulfate polyacrylamide gel electrophoresis (SDS-PAGE); protein kinase inhibitor a (PKI-a); cAMP response element-binding protein 
(CREB); Ras homolog gene family, member A (RhoA); Arginase 1 (Arg1); guanosine triphosphate (GTP); mutant (MUT); wild type (WT); human embryonic kidney (HEK); Neurofilament 200 (NF200); 4',6-diamidino-2-phenylindole (DAPI); Somatosensory evoked potentials (SSEP); miR (microRNA); Tape Removal Test (TRT); Rho-associated protein kinase (ROCK); Alzheimer's Disease (AD); Parkinson's disease (PD); blood spinal cord barrier (BSCB); blood brain barrier (BBB); Glutathione (GSH); neural precursor cells (NPC).

\section{Acknowledgements}

This work was supported by the General Program of Natural Science Foundation of Hebei Province of China (H2017101030), the Medical Science and Technology Youth Cultivation Project of the Chinese People's Liberation Army (16QNP074, 13QNP017), the Research and Development of Science and Technology Program Supported by Chengde Government (201606A062, 201701A125, 201701A127), the Beijing Municipal Education Commission General Project (KM201710025028), the Capital characteristic project (Z161100000116064), State Key Program of National Natural Science Foundation of China (81330042), International Cooperation Program of National Natural Science Foundation of China (81620108018), the National Natural Science Foundation of China (81472070, 81772342) and Key Program of Medical Science Foundation of Hebei province of China (20190189).

\section{Author Contributions}

Conceptualization, GXL, NGZ, FSQ and CXM; Methodology, WZJ; Software, XZW; Validation, WX, and YM; Formal Analysis, TW; Investigation, ZZ; ZL.; Data Curation, WX; Writing-Original Draft Preparation, LB, YX and WZJ; Writing-Review \& Editing, WTY and CCJ; Visualization, CCJ; Supervision, GXL, NGZ, FSQ, CXM; Project Administration, WTY; Funding Acquisition, WTY, CCJ, FSQ and CXM. All authors read and approved the final manuscript.

\section{Ethics approval and consent to participate}

All protocols were approved by the Animal Ethics Committee of the 266th Hospital of the Chinese People's Liberation Army (Approval No. 20160134). All authors consented to participate in this study.

\section{Competing Interests}

The authors have declared that no competing interest exists.

\section{References}

1. Hoschouer EL, Finseth T, Flinn S, et al. Sensory stimulation prior to spinal cord injury induces post-injury dysesthesia in mice. J Neurotrauma. 2010; 27: 777-87.

2. Hongyun Huang HSS, Lin Chen, Ali Otom, Ziad M. Al Zoubi, Hooshang Saberi, Dafin F. Muresanu, Xijing He. Review of clinical neurorestorative strategies for spinal cord injury: Exploring history and latest progresses. Journal of Neurorestoratology. 2018; 6: 171-8.

3. Kang Y, Ding H, Zhou H, et al. Epidemiology of worldwide spinal cord injury: a literature review. Epidemiology of worldwide spinal cord injury: a literature review. 2018; 6: 1-9.

4. Wang T, Yuan W, Liu Y, et al. The role of the JAK-STAT pathway in neural stem cells, neural progenitor cells and reactive astrocytes after spinal cord injury. Biomed Rep. 2015; 3: 141-6.

5. Hao J, Li B, Duan HQ, et al. Mechanisms underlying the promotion of functional recovery by deferoxamine after spinal cord injury in rats. Neural Regen Res. 2017; 12: 959-68.

6. Wang W, Wang F, Liu J, et al. SNAP25 ameliorates sensory deficit in rats with spinal cord transection. Mol Neurobiol. 2014; 50: 290-304.

7. Richards JS, Hirt M, Melamed L. Spinal cord injury: a sensory restriction perspective. Archives of physical medicine and rehabilitation. 1982; 63: 195-9.

8. Krishnan KR, Glass CA, Turner SM, et al. Perceptual deprivation in the acute phase of spinal injury rehabilitation. The Journal of the American Paraplegia Society. 1992; 15: 60-5.

9. Ahmed Z, Douglas MR, Read ML, et al. Citron kinase regulates axon growth through a pathway that converges on cofilin downstream of RhoA. Neurobiol Dis. 2011; 41: 421-9.

10. Richardson PM, Issa VMK. Peripheral injury enhances central regeneration of primary sensory neurones. Nature. 1984; 309: 791-3

11. Wang $\mathrm{T}$, Liu $\mathrm{Y}, \mathrm{Yuan} \mathrm{W}$, et al. Identification of microRNAome in rat bladder reveals miR-1949 as a potential inducer of bladder cancer following spinal cord injury. Molecular Medicine Reports. 2015; 12: 2849-57.

12. Liang $\mathrm{Z}, \mathrm{X}_{\mathrm{i}} \mathrm{Y}$. MicroRNAs mediate therapeutic and preventive effects of natural agents in breast cancer. Chinese journal of natural medicines. 2016; 14: 881-7.

13. Wang $\mathrm{T}$, Liu $\mathrm{Y}$, Yuan $\mathrm{W}$, et al. Identification of microRNAome in rat bladder reveals miR-1949 as a potential inducer of bladder cancer following spinal cord injury. Mol Med Rep. 2015; 12: 2849-57.

14. Wang T, Yuan W, Liu Y, et al. miR-142-3p is a Potential Therapeutic Target for Sensory Function Recovery of Spinal Cord Injury. Med Sci Monit. 2015; 21: 2553-6.

15. Zhou S, Yu B, Qian T, et al. Early changes of microRNAs expression in the dorsal root ganglia following rat sciatic nerve transection. Neurosci Lett. 2011; 494: 89-93.

16. Wang T, Li B, Wang Z, et al. Sorafenib promotes sensory conduction function recovery via miR-142-3p/AC9/cAMP axis post dorsal column injury. Neuropharmacology. 2019; 148: 347-57.

17. Wang Z, Yuan W, Li B, et al. PEITC Promotes Neurite Growth in Primary Sensory Neurons via the miR-17-5p/STAT3/GAP-43 Axis. J Drug Target. 2018: 1-39.

18. Wang T, Li B, Yuan X, et al. MiR-20a Plays a Key Regulatory Role in the Repair of Spinal Cord Dorsal Column Lesion via PDZ-RhoGEF/RhoA/GAP43 Axis in Rat. Cell Mol Neurobiol. 2019; 39: 87-98.

19. Ghorpade DS, Leyland R, Kurowska-Stolarska M, et al. MicroRNA-155 is required for Mycobacterium bovis BCG-mediated apoptosis of macrophages. Mol Cell Biol. 2012; 32: 2239-53.

20. Lee HJ, Lee EJ, Seo M. Galpha12 Protects Vascular Endothelial Cells from Serum Withdrawal-Induced Apoptosis through Regulation of miR-155. Yonsei medical journal. 2016; 57: 247-53.

21. Harrison EB, Emanuel K, Lamberty BG, et al. Induction of miR-155 after Brain Injury Promotes Type 1 Interferon and has a Neuroprotective Effect. Front Mol Neurosci. 2017; 10: 228.

22. Fassi Fehri L, Koch M, Belogolova E, et al. Helicobacter pylori induces miR-155 in T cells in a cAMP-Foxp3-dependent manner. PLoS One. 2010; 5: e9500.

23. Dalton GD, Dewey WL. Protein kinase inhibitor peptide (PKI): a family of endogenous neuropeptides that modulate neuronal cAMP-dependent protein kinase function. Neuropeptides. 2006; 40: 23-34.

24. Qiu J, Cai D, Dai H, et al. Spinal axon regeneration induced by elevation of cyclic AMP. Neuron. 2002; 34: 895-903.

25. Qiu J, Cafferty WB, McMahon SB, et al. Conditioning injury-induced spinal axon regeneration requires signal transducer and activator of transcription 3 activation. The Journal of neuroscience : the official journal of the Society for Neuroscience. 2005; 25: 1645-53.

26. Neumann S, Woolf CJ. Regeneration of dorsal column fibers into and beyond the lesion site following adult spinal cord injury. Neuron. 1999; 23: 83-91.

27. Christie KJ, Webber CA, Martinez JA, et al. PTEN inhibition to facilitate intrinsic regenerative outgrowth of adult peripheral axons. J Neurosci. 2010; 30: 9306-15.

28. Jiang J, Song Z, Zhang L. miR-155-5p Promotes Progression of Acute Respiratory Distress Syndrome by Inhibiting Differentiation of Bone Marrow Mesenchymal Stem Cells to Alveolar Type II Epithelial Cells. Med Sci Monit. 2018; $24: 4330-8$ 
29. Han Q, Lin Q, Huang P, et al. Microglia-derived IL-1beta contributes to axon development disorders and synaptic deficit through p38-MAPK signal pathway in septic neonatal rats. J Neuroinflammation. 2017; 14: 52

30. Zhang L, Yue Y, Ouyang M, et al. The Effects of IGF-1 on TNF-alpha-Treated DRG Neurons by Modulating ATF3 and GAP-43 Expression via PI3K/Akt/S6K Signaling Pathway. Neurochem Res. 2017; 42: 1403-21.

31. Tsai SY, Yang LY, Wu CH, et al. Injury-induced Janus kinase/protein kinase C-dependent phosphorylation of growth. Journal of Neuroscience Research. 2010; 85: 321-31.

32. Song J, Cheon SY, Lee WT, et al. PKA Inhibitor H89 (N-[2-p-bromocinnamylamino-ethyl]-5-isoquinolinesulfonamide) Attenuates Synaptic Dysfunction and Neuronal Cell Death following Ischemic Injury. Neural Plast. 2015; 2015: 374520.

33. Yamashita $T$, Tohyama $M$. The $p 75$ receptor acts as a displacement factor that releases Rho from Rho-GDI. Nat Neurosci. 2003; 6: 461-7.

34. Zhao X, Chen C, Wei Y, et al. Novel mutations of COL4A3, COL4A4, and COL4A5 genes in Chinese patients with Alport Syndrome using next generation sequence technique. Molecular genetics \& genomic medicine. 2019: e653.

35. Ren JL, Yu QX, Liang WC, et al. Green tea extract attenuates LPS-induced retinal inflammation in rats. Sci Rep. 2018; 8: 429

36. Zhou $\mathrm{H}, \mathrm{Li} \mathrm{X}, \mathrm{Wu} \mathrm{Q}$, et al. shRNA against PTEN promotes neurite outgrowth of cortical neurons and functional recovery in spinal cord contusion rats. Regen Med. 2015; 10: 411-29.

37. Mason MR, Ehlert EM, Eggers R, et al. Comparison of AAV serotypes for gene delivery to dorsal root ganglion neurons. Molecular Therapy the Journal of the American Society of Gene Therapy. 2010; 18: 715 .

38. Han $\mathrm{X}$, Yang $\mathrm{N}, \mathrm{Xu} \mathrm{Y}$, et al. Simvastatin treatment improves functional recovery after experimental spinal cord injury by upregulating the expression of BDNF and GDNF. Neurosci Lett. 2011; 487: 255-9.

39. Basso DM, Beattie MS, Bresnahan JC. A sensitive and reliable locomotor rating scale for open field testing in rats. J Neurotrauma. 1995; 12: 1-21.

40. Moreno-Flores MT, Bradbury EJ, Martin-Bermejo MJ, et al. A clonal cell line from immortalized olfactory ensheathing glia promotes functional recovery in the injured spinal cord. Mol Ther. 2006; 13: 598-608

41. Fagoe ND, Attwell CL, Eggers R, et al. Evaluation of Five Tests for Sensitivity to Functional Deficits following Cervical or Thoracic Dorsal Column Transection in the Rat. PLoS One. 2016; 11: e0150141.

42. Harrington AW, Li QM, Tep C, et al. The Role of Kalirin9 in p75/Nogo Receptor-mediated RhoA Activation in Cerebellar Granule Neurons. Journal of Biological Chemistry. 2008; 283: 24690-7.

43. Rafferty BJ, Unger BL, Perey AC, et al. A novel role for the Rho-associated kinase, ROCK, in IL-1-stimulated intestinal epithelial cell responses. Cell Immunol. 2012; 280: 148-55.

44. Hung CN, Huang HP, Wang CJ, et al. Sulforaphane inhibits TNF-alpha-induced adhesion molecule expression through the Rho A/ROCK/NF-kappaB signaling pathway. Journal of medicinal food. 2014; 17 : 1095-102.

45. Resnick DK, Schmitt C, Miranpuri GS, et al. Molecular evidence of repair and plasticity following spinal cord injury. Neuroreport. 2004; 15: 837-9.

46. Richards JS, Hirt M, Melamed L. Spinal cord injury: a sensory restriction perspective. Archives of physical medicine and rehabilitation. 1982; 63: 195-9.

47. Krishnan KR, Glass CA, Turner SM, et al. Perceptual deprivation in the acute phase of spinal injury rehabilitation. Journal of the American Paraplegia Society. $1992 ; 15: 60-5$

48. Ao Q, Wang AJ, Chen GQ, et al. Combined transplantation of neural stem cells and olfactory ensheathing cells for the repair of spinal cord injuries. Med Hypotheses. 2007; 69: 1234-7.

49. Gao L, Dai C, Feng Z, et al. MiR-137 inhibited inflammatory response and apoptosis after spinal cord injury via targeting of MK2. Journal of Cellular Biochemistry. 2018; 119.

50. Hebert SS, Horre K, Nicolai L, et al. MicroRNA regulation of Alzheimer's Amyloid precursor protein expression. Neurobiol Dis. 2009; 33: 422-8.

51. Wang E, Cambi F. MicroRNA expression in mouse oligodendrocytes and regulation of proteolipid protein gene expression. Journal of Neuroscience Research. 2012; 90: 1701-12.

52. Janssen HL, Reesink HW, Lawitz EJ, et al. Treatment of HCV infection by targeting microRNA. N Engl J Med. 2013; 368: 1685-94.

53. Abel T, Nguyen PV, Barad M, et al. Genetic demonstration of a role for PKA in the late phase of LTP and in hippocampus-based long-term memory. Cell. 1997; 88: 615-26

54. De LL, Criado JR, Rivera $\mathrm{S}$, et al. Endogenous protein kinase A inhibitor (PKIalpha) modulates synaptic activity. Journal of Neuroscience Research. 1998; 53: 269.

55. Li X, Lyons AB, Woods GM, et al. The absence of TNF permits myeloid Arginase 1 expression in experimental L. monocytogenes infection. Immunobiology. 2017; 222: 913-7.

56. Wu DM, Wen X, Han XR, et al. MiR-142-3p Enhances Cell Viability and Inhibits Apoptosis by Targeting CDKN1B and TIMP3 Following Sciatic Nerve Injury. Cell Physiol Biochem. 2018; 46: 2347-57.

57. Lee YY, Yarmishyn AA, Wang ML, et al. MicroRNA-142-3p is involved in regulation of MGMT expression in glioblastoma cells. Cancer management and research. 2018; 10: 775-85.
58. Oh Y, Park J, Kim JI, et al Lin28B and miR-142-3p regulate neuronal differentiation by modulating Staufen1 expression. Cell Death Differ. 2018; 25: $432-43$.

59. Mandolesi G, De Vito F, Musella A, et al. miR-142-3p Is a Key Regulator of IL-1beta-Dependent Synaptopathy in Neuroinflammation. J Neurosci. 2017; 37: 546-61.

60. Huang LG, Zou J, Lu QC. Silencing rno-miR-155-5p in rat temporal lobe epilepsy model reduces pathophysiological features and cell apoptosis by activating Sestrin-3. Brain Res. 2018; 1689: 109-22

61. Gao W, Bi Y, Ding L, et al. SSa ameliorates the Glu uptaking capacity of astrocytes in epilepsy via AP-1/miR-155/GLAST. Biochem Biophys Res Commun. 2017; 493: 1329-35

62. Gaudet AD, Mandrekar-Colucci S, Hall JC, et al. miR-155 Deletion in Mice Overcomes Neuron-Intrinsic and Neuron-Extrinsic Barriers to Spinal Cord Repair. J Neurosci. 2016; 36: 8516-32.

63. Mycko MP, Cichalewska M, Cwiklinska H, et al. miR-155-3p Drives the Development of Autoimmune Demyelination by Regulation of Heat Shock Protein 40. J Neurosci. 2015; 35: 16504-15.

64. Gamdzyk M, Doycheva DM, Malaguit J, et al. Role of PPAR-beta/delta/miR-17/TXNIP pathway in neuronal apoptosis after neonatal hypoxic-ischemic injury in rats. Neuropharmacology. 2018; 140: $150-61$.

65. Chen D, Dixon BJ, Doycheva DM, et al. IRE1alpha inhibition decreased TXNIP/NLRP3 inflammasome activation through miR-17-5p after neonatal hypoxic-ischemic brain injury in rats. J Neuroinflammation. 2018; 15: 32.

66. Hong $\mathrm{P}$, Jiang $\mathrm{M}, \mathrm{Li} \mathrm{H}$. Functional requirement of dicer1 and miR-17-5p in reactive astrocyte proliferation after spinal cord injury in the mouse. Glia. 2014; 62: 2044-60.

67. Mao S, Li H, Sun Q, et al. miR-17 regulates the proliferation and differentiation of the neural precursor cells during mouse corticogenesis. The FEBS journal. 2014; 281: 1144-58.

68. Huang ZZ, Wei JY, Ou-Yang HD, et al. mir-500-Mediated GAD67 Downregulation Contributes to Neuropathic Pain. J Neurosci. 2016; 36: 6321-31.

69. Gan J, Cai O, Ou Y, et al. miR-96 attenuates status epilepticus-induced brain injury by directly targeting Atg7 and Atg16L1. Sci Rep. 2017; 7: 10270.

70. Bian $\mathrm{H}$, Shan $\mathrm{H}$, Chen $\mathrm{T}$. Resveratrol ameliorates hypoxia/ischemia-induced brain injury in the neonatal rat via the miR-96/Bax axis. Child's nervous system : ChNS : official journal of the International Society for Pediatric Neurosurgery. 2017; 33: 1937-45.

71. Kinoshita C, Aoyama K, Matsumura N, et al. Rhythmic oscillations of the microRNA miR-96-5p play a neuroprotective role by indirectly regulating glutathione levels. Nat Commun. 2014; 5: 3823

72. Du ZW, Ma LX, Phillips C, et al. miR-200 and miR-96 families repress neural induction from human embryonic stem cells. Development. 2013; 140: 2611-8.

73. Kouhkan F, Mobarra N, Soufi-Zomorrod M, et al. MicroRNA-129-1 acts as tumour suppressor and induces cell cycle arrest of GBM cancer cells through targeting IGF2BP3 and MAPK1. J Med Genet. 2016; 53: 24-33.

74. He J, Zhao J, Peng X, et al. Molecular Mechanism of MiR-136-5p Targeting NF-kappaB/A20 in the IL-17-Mediated Inflammatory Response after Spinal Cord Injury. Cell Physiol Biochem. 2017; 44: 1224-41.

75. Gong G, An F, Wang Y, et al. miR-15b represses BACE1 expression in sporadic Alzheimer's disease. Oncotarget. 2017; 8: 91551-7.

76. Shi H, Sun BL, Zhang J, et al. miR-15b suppression of Bcl-2 contributes to cerebral ischemic injury and is reversed by sevoflurane preconditioning. CNS Neurol Disord Drug Targets. 2013; 12: 381-91.

77. Pang C, Guan Y, Zhao K, et al. Up-regulation of microRNA-15b correlates with unfavorable prognosis and malignant progression of human glioma. Int J Clin Exp Pathol. 2015; 8: 4943-52.

78. Lv X, Jiang H, Liu Y, et al. MicroRNA-15b promotes neurogenesis and inhibits neural progenitor proliferation by directly repressing TET3 during early neocortical development. EMBO Rep. 2014; 15: 1305-14.

79. Zhi F, Wang Q, Deng D, et al. MiR-181b-5p downregulates NOVA1 to suppress proliferation, migration and invasion and promote apoptosis in astrocytoma. PLoS One. 2014; 9: e109124.

80. Zhang T, Ni S, Luo Z, et al. The protective effect of microRNA-21 in neurons after spinal cord injury. Spinal Cord. 2018.

81. Strickland IT, Richards L, Holmes FE, et al. Axotomy-induced miR-21 promotes axon growth in adult dorsal root ganglion neurons. PLoS One. 2011; 6: e23423

82. Xie W, Yang SY, Zhang Q, et al. Knockdown of MicroRNA-21 Promotes Neurological Recovery After Acute Spinal Cord Injury. Neurochem Res. 2018.

83. Li XQ, Fang B, Tan WF, et al. miR-320a affects spinal cord edema through negatively regulating aquaporin-1 of blood-spinal cord barrier during bimodal stage after ischemia reperfusion injury in rats. BMC Neurosci. 2016; 17: 10 .

84. Aung LL, Mouradian MM, Dhib-Jalbut S, et al. MMP-9 expression is increased in $\mathrm{B}$ lymphocytes during multiple sclerosis exacerbation and is regulated by microRNA-320a. J Neuroimmunol. 2015. 278: 185-9.

85. Kabaria $\mathrm{S}$, Choi DC, Chaudhuri AD, et al. Inhibition of miR-34b and miR-34c enhances alpha-synuclein expression in Parkinson's disease. FEBS Lett. 2015; 589: 319-25.

86. Cao $\mathrm{SE}$, Tian $\mathrm{J}$, Chen $\mathrm{S}$, et al. Role of $\mathrm{miR}-34 \mathrm{c}$ in ketamine-induced neurotoxicity in neonatal mice hippocampus. Cell Biol Int. 2015; 39: 164-8. 
87. Wu Z, Wu Y, Tian $Y$, et al. Differential effects of miR-34c-3p and miR-34c-5p on the proliferation, apoptosis and invasion of glioma cells. Oncol Lett. 2013; 6: $1447-52$.

88. Zovoilis A, Agbemenyah HY, Agis-Balboa RC, et al. microRNA-34c is a novel target to treat dementias. Embo j. 2011; 30: 4299-308.

89. Minones-Moyano E, Porta S, Escaramis G, et al. MicroRNA profiling of Parkinson's disease brains identifies early downregulation of miR-34b/c which modulate mitochondrial function. Hum Mol Genet. 2011; 20: 3067-78.

90. Ross SP, Baker KE, Fisher A, et al. miRNA-431 Prevents Amyloid-beta-Induced Synapse Loss in Neuronal Cell Culture Model of Alzheimer's Disease by Silencing Kremen1. Front Cell Neurosci. 2018; 12: 87.

91. Wertz MH, Winden K, Neveu $P$, et al. Cell-type-specific miR-431 dysregulation in a motor neuron model of spinal muscular atrophy. Hum Mol Genet. 2016; 25: 2168-81. 